\title{
Cranioesophageal Pythiosis in a Horse
}

\author{
Rabeche Schmith', Vírgilio Zoppi Lemos', Diogo Almeida Rondon', Luiz Alexandre Moscon', \\ Ana Lucia Schild², Elisa Simone Viégas Sallis², Daniela Isabel Brayer Pereira ${ }^{3}$ \& Clairton Marcolongo Pereira'
}

\begin{abstract}
Background: Pythiosis is a chronic inflammatory disease that is caused by oomycete Pythium insidiosum. This illness affects several species including humans and horses. Equine is the most affected species, having no predisposition for breed, gender, or age. It is usually shown in cutaneous and subcutaneous forms, and the lesions, which grow quickly and are hard to treat, are located mainly in the extremities. The diagnosis is made via epidemiology, clinical signs, and macroscopic and microscopic aspects of the lesion. This study describes a case of cranioesophageal pythiosis in a horse, examining the epidemiological, clinical and pathological characteristics.
\end{abstract}

Case: A 12-year-old male quarter horse, weighing $515 \mathrm{~kg}$ was taken to the Veterinary Hospital at the University Center of Espírito Santo (UNESC). The horse had an increase in volume in the cranioesophageal region, coughing, difficulty breathing, and a runny nose. On clinical examination, the horse showed an enlargement in the submandibular and retropharyngeal lymph nodes, subcutaneous edema in the larynx region, and a temperature of $38.2^{\circ} \mathrm{C}$. According to the owner, cough was recurrent and had lasted about 12 months even after treatment with different kinds of antimicrobials. On radiographic exam, there was a marked decrease in the tracheal lumen and increased soft tissue radiopacity in the region adjacent to the narrowing. The animal was taken to surgery to remove the mass, but he died because of complications during surgery. The animal's owner did not allow necropsy, but a fragment of the mass in the cranioesophageal was removed and sent for histological examination. The fragment was fixed in $10 \%$ formalin and processed using routine histological analysis. Macroscopically, the mass was light yellowish and ulcerated, and it measured $7.0 \times 5.0 \times 5.0 \mathrm{~cm}$. In the middle of the ulcerated areas, there were yellow and firm granular structures that were consistent with kunkers. Histologically, extending from the tracheal adventitia to the thyroid, there was a large number of lymphocytes, macrophages, neutrophils, eosinophils, and multinucleated cells (foreign body type) and a well-defined focus of coagulative necrosis, which was surrounded by a thin border of macrophages. Within the necrotic areas, there were negative images of tubuliform hyphae. Grocott's silver methanamine staining showed hyphae that had irregular branches, rare septa, smooth and parallel walls, and was impregnated by silver. Histological sections of the mass were subjected to immunohistochemistry. Hyphae were positive for Pythium insidiosum.

Discussion: The diagnosis of pythiosis was based on macroscopic, histological findings and positive immunostaining for Pythium insidiosum. This report shows the unusual location of the disease in the horse, which made the clinical diagnosis of the disease complex. Extracutaneous forms of pythiosis in horses are less frequent than cutaneous forms. The etiopathogenesis of these forms is still unclear, but it has been suggested that previous lesions in the intestinal mucosa caused by plant material or pathogens may be predisposing factors for the appearance of the enteric form of the disease. It was not possible to observe if the animal's other organs were affected because a necropsy could not be performed. The agent probably penetrated the esophageal epithelium and spread throughout the trachea and thyroid, but its origin cannot be determined. The radiographic findings in this study are compatible with neoplasms. However, inflammatory processes such as those caused by pythiosis should be included in the differential diagnosis of horses with swelling in the cranial portion of the esophagus.

Keywords: equine, Pythium insidiosum, cranioesophageal region. 


\section{INTRODUCTION}

Pythiosis is a chronic inflammatory disease that is caused by the aquatic oomycete Pythium insidiosum [14]. Pythium species are plant pathogens, but they occasionally cause disease in several animal species [8].

In nature, $P$. insidiosum performs its biological cycle in stagnant waters. In these environments, the microorganism colonizes aquatic plants for their development and asexual reproduction with the formation of infectious zoospores. Susceptible hosts acquire infection upon contact with contaminated environments [9].

Pythiosis occurs in different regions around the world [3]. In Brazil, the disease in horses is considered to be endemic in the Brazilian Pantanal [14] and Rio Grande do Sul [7].

Horses are most affected by the disease, and there is no predisposition for breed, sex, or age. The disease usually presents in cutaneous and subcutaneous forms [3], but effects on other organs have been described $[5,11,14]$.

The lesions are characterized by ulcerated masses and varied sizes, which drain serosanguinous fluid. These lesions are usually located on parts of the body that are in contact with water. In these masses, there are light yellowish concretions of necrotic material called kunkers [3], and microscopically, areas of necrosis are observed, with eosinophils, collagenolysis, and fibrosis, and Splendore-Hoeppli reaction involving hyphae [8], which are better visualized with silver-based dyes [8]. Molecular and immunohistochemical methods can confirm the diagnosis [8].

The aim was to describe a case of pythiosis in the cranioesophageal region of a horse, and to address its epidemiological, clinical, and pathological characteristics.

\section{CASE}

A male 12-year-old quarter horse weighing 515 $\mathrm{kg}$ was taken to the Veterinary Hospital of the University Center of Espírito Santo (UNESC) and presented with an increased volume in the cranioesophageal region, coughing, difficulty breathing, and runny nose. Clinical examination showed enlarged submandibular and retropharyngeal lymph nodes and subcutaneous edema in the larynx region, and the temperature was $38.2^{\circ} \mathrm{C}$. The owner indicated that the cough was recurrent and had lasted about 12 months even with different antimicrobial treatments. This horse lived in a stable and was only used for racing and training. The animal ate and drank in the stall. The water came from an artesian well on the property.

A radiograph showed a marked decrease in the tracheal lumen with increased soft tissue radiopacity in the region that was adjacent to the narrowing (Figure 1A).

The full-blood count (FBC) and biochemical profile did not show any alterations. The leukogram had leukocytosis resulting from neutrophilia.

The animal was taken to surgery to remove the mass, but died because of complications during surgery. The animal's owner did not allow necropsy, but a fragment of the cranioesophageal mass was removed and sent for histological examination. The fragment was fixed in $10 \%$ formalin and processed routinely for histological analysis and stained with hematoxylin and eosin ${ }^{1}$ (HE). Histological sections were also subjected to special histochemical staining using Grocott's methenamine silver nitrate ${ }^{1}$.

Macroscopically, the mass was light yellowish, ulcerated, and measured $7.0 \times 5.0 \times 5.0 \mathrm{~cm}$. In the middle of the ulcerated areas, there were yellow and firm granular structures, which were consistent with kunkers (Figure 1B). Histologically, extending from the trachea's adventitia to the thyroid, there were large numbers of lymphocytes, macrophages, neutrophils, eosinophils, and multinucleated cells (foreign body type) and a well-defined focus of coagulative necrosis, which was surrounded by a thin border of macrophages (Figures 1C). Within the necrotic areas, there were negative images of tubuliform hyphae (Figure 1C - insert). Grocott's silver methanamine staining showed that hyphae had irregular branches, rare septa, smooth and parallel walls, and they were impregnated by silver (Figure 2A).

Histological sections of the mass were subjected to immunohistochemistry (IHC) using anti- $P$. insidiosum antibody (non-commercial) at a dilution of 1:500 [13]. It was incubated at $37^{\circ} \mathrm{C}$ for $2 \mathrm{~h}$ and linked to the streptavidin-peroxidase complex [9]. The chromogen used was 3,3'-diaminobenzidine tetrachloride $(\mathrm{DAB})^{2}[9]$. The cuts were stained with Harris' hematoxylin $^{3}$ [13]. The hyphae were positively marked for P. insidiosum (Figure 2B).

\section{DISCUSSION}

The diagnosis of pythiosis was based on macroscopic and histological findings and positive immunostaining for $P$. insidiosum. The location of 


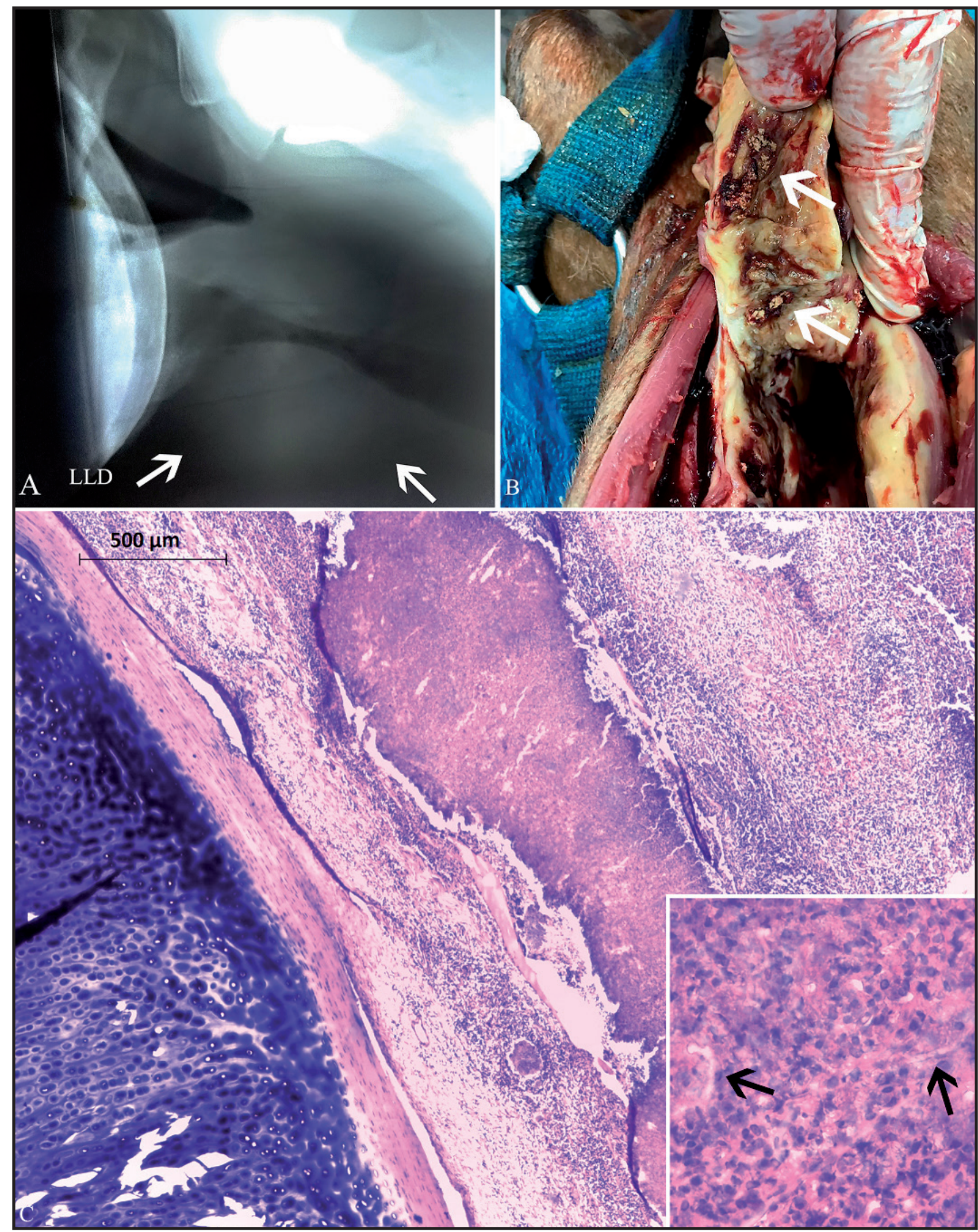

Figure 1. Cranioesophageal pythiosis in a horse. A- Right lateral-lateral projection. There is a marked decrease in the tracheal lumen with increased soft tissue radiopacity in the region adjacent to the narrowing (white arrows). B- A light yellowish, ulcerated mass that was removed from the cranioesophageal region can be observed with firm yellow granular structures, which are consistent with kunkers. C- A well-defined focus of coagulative necrosis extending from the trachea's adventitia. Insert. Presence of negative images of tubiliform hyphae (black arrows) [HE; 40× magnification]. 


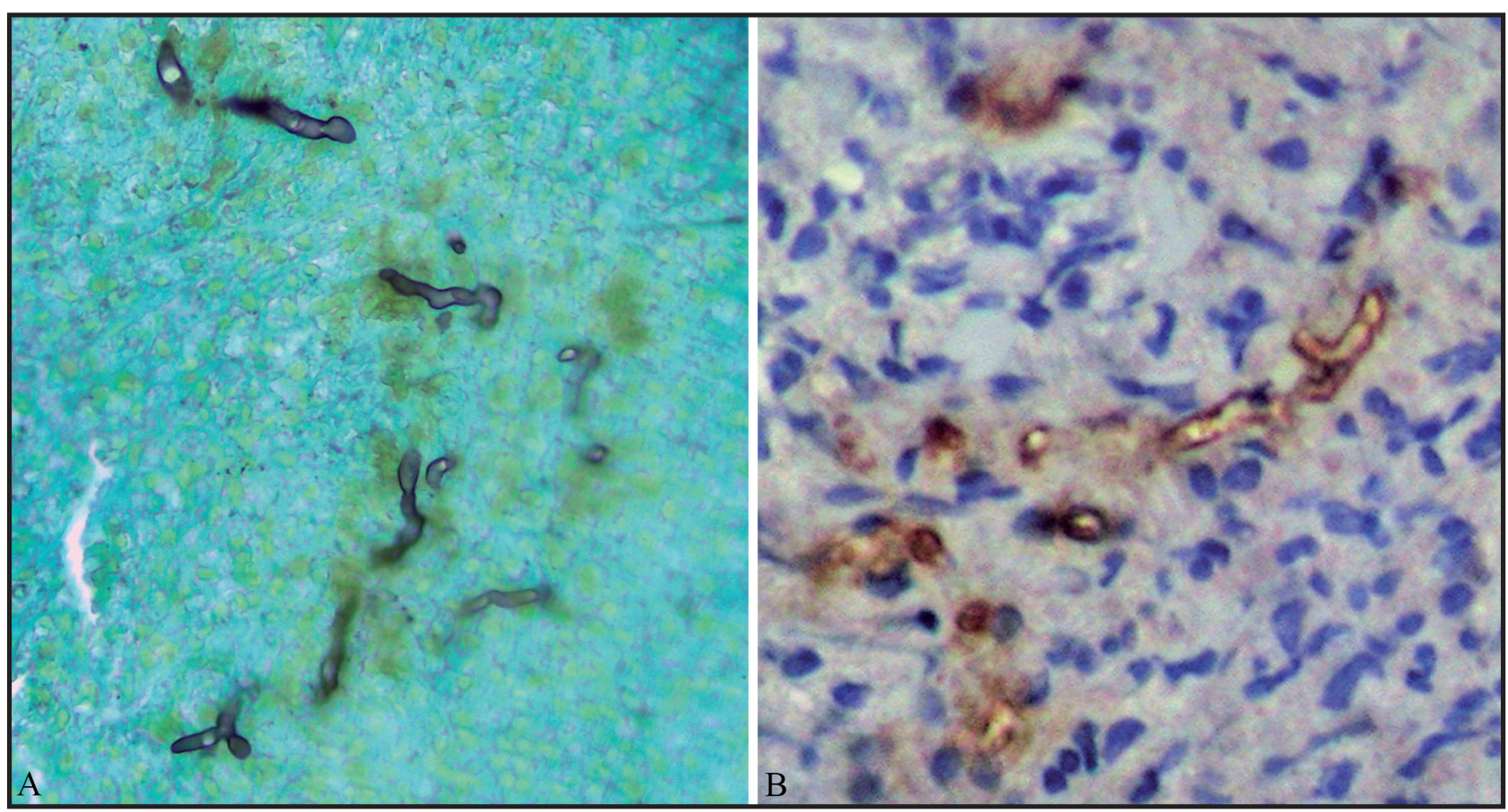

Figure 2. Cranioesophageal pythiosis in a horse. A- Irregular branched hyphae are observed, with rare septations and smooth and parallel walls [Grocott; 40× magnification]. B- Positive immunolabelling for P. insidiosum [counterstaining with Harris' hematoxylin; 40x magnification].

the disease in the horse was unusual, which made the clinical diagnosis of the disease complex because the volume increase and radiographic changes were suggestive of neoplasm. Extracutaneous forms of pythiosis in horses are less frequent than cutaneous forms, and the etiopathogenesis of these forms are not well understood. It has been suggested that previous lesions in the gastrointestinal tract mucosa caused by plant material or pathogens may be predisposing factors for the appearance of the extracutaneous form of pythiosis $[10,11]$. In addition, there are reports that the disease can occur because of active penetration of the agent into the tissues [1]. The agent likely penetrated the esophageal epithelium directly or by some injury that was caused by coarse food, and it spread around the trachea and thyroid. However, its precise origin cannot be determined.

In this study, it was not possible to observe whether the animal's other organs were affected because necropsy could not be performed. Extracutaneous pythiosis has been described in other species including lung injury in jaguars [2] and canines [6] and sublingual pythiosis in felines [4].
The cough and breathing difficulties that were observed in this horse probably resulted from compression of the trachea that was caused by the proliferative inflammatory lesion. A similar case was observed in a horse with severe breathing difficulties caused by pythiosis in Rio Grande do Sul. In this case, the disease affected the soft palate and caused nasopharyngeal obliteration, which resulted in breathing difficulties [12].

The radiographic findings in this study are compatible with neoplasm. However, inflammatory processes such as those caused by pythiosis should be included in the differential diagnosis of horses with swelling in the cranial portion of the esophagus.

\section{MANUFACTURERS}

${ }^{1}$ Sigma-Aldrich Inc. Milwaukee, WI, U.S.A.

${ }^{2}$ Dako an Agilent Technologies Company. Carpinteria, CA, U.S.A.

${ }^{3}$ WCor Corantes Comércio e Serviços Eireli. Guarulhos, SP, Brazil.

Acknowledgements. This work was supported by Fundação de Amparo à Pesquisa Inovação do Espírito Santo - FAPES.

Declaration of interest. The authors report no conflicts of interest. The authors alone are responsible for the content and writing of the paper. 


\section{REFERENCES}

1 Brown C.C. \& Roberts E.D. 1988. Intestinal pythiosis in a horse. Australian Veterinary Journal. 65(3): 88-89.

2 Camus A.C., Grooters A.M. \& Aquilar R.F. 2004. Granulomatous pneumonia caused by Pythium insidiosum in a central American jaguar, Panthera onca. Journal of Veterinary Diagnostic Investigation. 16(6): 567-571.

3 Fonseca A.O., Ávila S.B., Nogueira C.E.W., Corrêa B.F., Souza J.S., Azevedo M.I. \& Pereira D.I.B. 2013. In vitro reproduction of the life cycle of Pythium insidiosum from Kunkers' equine and their role in the epidemiology of Pythiosis. Mycopathologia. 177(1-2): 123-127.

4 Fortin J.S., Calcutt M.J. \& Kim D.Y. 2017. Sublingual pythiosis in a cat. Acta Veterinaria Scandinavica. $59(1): 63$. 5 Goad M.E.P. 1984. Pulmonary pythiosis in a horse. Veterinary Pathology. 21(2): 261-262.

6 Kepler D., Cole R., Lee-Fowler T., Koehler J., Shrader S. \& NewtonJ. 2019. Pulmonary pythiosis in a canine patient. Veterinary Radiology \& Ultrasound. 60(2): E20-E23.

7 Marcolongo-Pereira C., Sallis E.S.V., Raffi M.B., Pereira D.I.B., Hinnah F.L., Coelho A.C.B. \& Schild A.L. 2012. Epidemiology of equine pythiosis in southern of Rio Grande do Sul State, Brazil. Pesquisa Veterinária Brasileira. 32(9): 865-868.

8 Martins T.B., Kommers G.D., Trost M.E., Inkelmann M.A., Fighera R.A. \& Schild A.L. 2012. A Comparative Study of the Histopathology and Immunohistochemistry of Pythiosis in Horses, Dogs and Cattle. Journal of Comparative Pathology. 146(2-3): 122-131.

9 Mendoza L., Ajello L. \& Mcginnis M.R. 1996. Infections caused by the oomycetous pathogen Pythium insidiosum. Journal de Mycologie Médicale. 6(4):151-164.

10 Morton L.D., Morton D.G., Baker G.J. \& Gelberg HB. 1991. Chronic eosinophilic enteritis attributed to Pythium sp. in a horse. Veterinary Pathology. 28(6): 542-544.

11 Purcell K.L., Johnson P.J., Kreeger J.M. \& Wilson D.A. 1994. Jejunal obstruction caused by a Pythium insidiosum granuloma in a mare. American Veterinary Medical Association. 205(2): 337-9.

12 Rossato C.K., Fiss L., Sperotto V.R., Cardona R.O.C. \& Silva R.B. 2018. Pythiosis with atypical location in the soft palate in a horse in Southern Brazil. Arquivo Brasileiro de Medicina Veterinária e Zootecnia. 70(2): 641-643.

13 Santos C.E.P., Ubiali D.G., Pescador C.A., Zanette R.A., Santurio J.M. \& Marques L.C. 2014. Epidemiological survey of equine pythiosis in the Brazilian Pantanal and nearby areas: Results of 76 cases. Journal of Equine Veterinary Science. 34(2): 270-274.

14 Souto E.P.F., Maia L.A., Olinda R.G., Galiza G.J.N., Kommers G.D., Miranda-Neto E.G. \& Riet-Correa F. 2016. Pythiosis in the nasal cavity of horses. Journal of Comparative Pathology. 155(2-3): 126-129.

http://seer.ufrgs.br/ActaScientiaeVeterinariae 